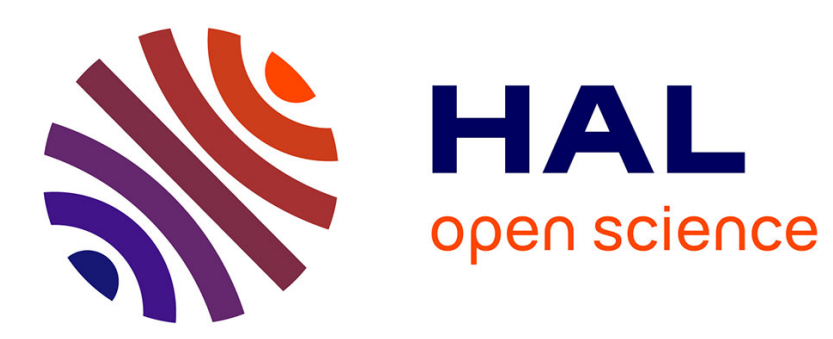

\title{
Comparison of Direct and Indirect Combustion Noise Mechanisms in a Model Combustor
}

\author{
Matthieu Leyko, Franck Nicoud, Thierry Poinsot
}

\section{To cite this version:}

Matthieu Leyko, Franck Nicoud, Thierry Poinsot. Comparison of Direct and Indirect Combustion Noise Mechanisms in a Model Combustor. AIAA Journal, 2009, 47 (11), pp.2709-2716. 10.2514/1.43729 . hal-00803808

\section{HAL Id: hal-00803808 https://hal.science/hal-00803808}

Submitted on 22 Mar 2013

HAL is a multi-disciplinary open access archive for the deposit and dissemination of scientific research documents, whether they are published or not. The documents may come from teaching and research institutions in France or abroad, or from public or private research centers.
L'archive ouverte pluridisciplinaire HAL, est destinée au dépôt et à la diffusion de documents scientifiques de niveau recherche, publiés ou non, émanant des établissements d'enseignement et de recherche français ou étrangers, des laboratoires publics ou privés. 


\title{
Comparison of direct and indirect combustion noise mechanisms in a model combustor
}

\author{
M. Leyko* \\ SNECMA, 77550 Moissy-Cramayel, France-CERFACS, 31057 Toulouse, France \\ F. Nicoud ${ }^{\dagger}$ \\ Université Montpellier II, 34095 Montpellier, France \\ T. Poinsot ${ }^{\ddagger}$ \\ Institut de Mécanique des Fluides de Toulouse, 31400 Toulouse, France
}

\begin{abstract}
Core-noise in aero-engines is due to two main mechanisms: direct combustion noise, which is generated by the unsteady expansion of burning gases and indirect combustion noise, which is due to the acceleration of entropy waves (temperature fluctuations generated by unsteady combustion) within the turbine stages. This paper shows how a simple burner model (a flame in a combustion chamber terminated by a nozzle) can be used to scale direct and indirect noise. This is done using an analytical formulation for waves generated by combustion and a numerical tool for waves transmission-generation in a nozzle. Numerical results for the nozzle verify and extend the analytical approach of Marble \& Candel. The analytical relations for the combustion and the nozzle provide simple scaling laws for direct and indirect noise ratio as a function of the Mach number in the combustion chamber and at the nozzle outlet.
\end{abstract}

\section{Nomenclature}

$\mathcal{A} \quad$ nozzle cross-section area, $m^{2}$

\footnotetext{
*PhD Student, email: leyko@cerfacs.fr.

${ }^{\dagger}$ Professor.

${ }^{\ddagger}$ Research Director, AIAA Associate Fellow.
} 


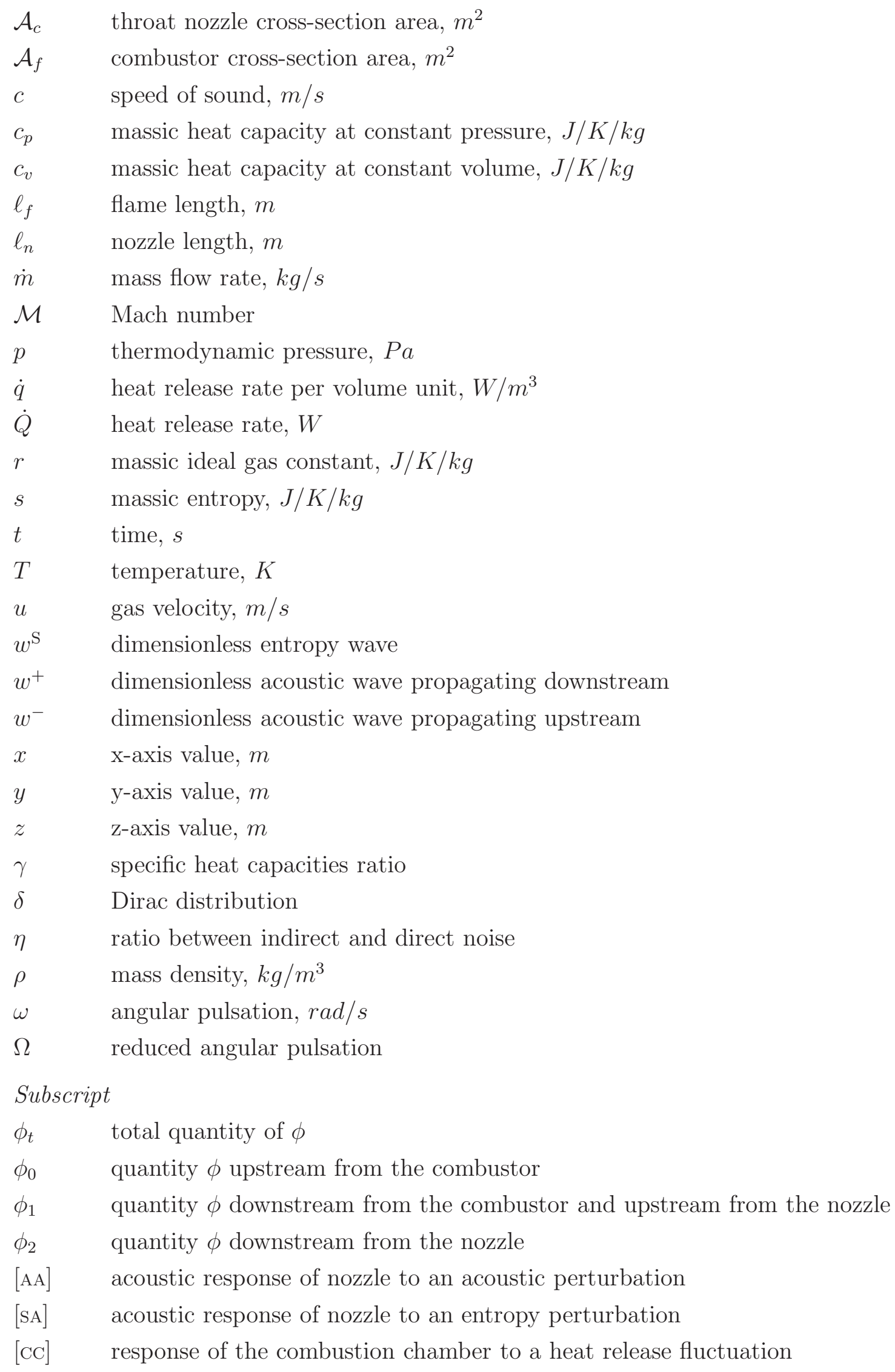




\section{Special notations}

$\bar{\phi} \quad$ temporal mean value of $\phi$

$\phi^{\prime} \quad$ temporal fluctuation value of $\phi$

PW $\{\phi\}$ Spectral power density of $\phi$ computed with Welch's method

\section{Introduction}

Over the last five decades, jet and external aerodynamic noises of aircraft have been substantially reduced. Further developments will be needed for modern aircraft design in order to meet the increasingly restrictive rules about noise reduction. While drastic reductions have already been achieved on fan and jet noise, the relative importance of other noise sources has increased and the contribution of these sources must be controlled if further global noise reduction is to be achieved. Among these sources, the noise created by the turbulent flame within the combustor is already identified as non-negligible at take-off, especially in the mid-frequency range. Two main mechanisms have been identified in the seventies regarding noise propagation and generation from the combustion chamber to the far field (Fig. 1):

- direct combustion noise: acoustic perturbations generated by the unsteady heat release from the turbulent flame propagate either upstream or downstream through the turbomachinery stages where they are distorted by the mean flow, diffracted and reflected by the solid walls within the diffuser, the distributer and the turbine and compressor blades.

- indirect combustion noise: entropy fluctuations generated within the combustion chambers (hot spots, imperfect mixing, etc.) propagate downstream and interact with accelerating mean flow. The cinematic and thermodynamic variables are strongly coupled when the flow is compressible, and thus fluctuations on the mass density generate perturbations on the others flow variables where the sharp flow variation through turbines stages occurs. Subsequent acoustic waves are generated and transmitted to the far field through the turbine stages.

Direct combustion noise is typically the main source of noise of a free flame. The acoustic radiation due to a turbulent flame has been theoretically treated by Bragg, ${ }^{1}$ Strahle, ${ }^{2}$ Hassan ${ }^{3}$ and others. More recently, Ihme ${ }^{4}$ computes successfully the sound emitted from a turbulent diffusion flame by combining Large Eddy Simulation and computational aeroacoustic method, providing precious informations about the combustion-generated noise. Experimental and theoretical work of Candel and co-authors ${ }^{5,6}$ about the noise generated by unsteady 
laminar flames, as well as the developments of Dowling in Crighton et al., ${ }^{7}$ and experimental investigations of Lieuwen and colleagues $^{8-10}$ about combustion-generated noise of turbulent flames, are also important source of informations for direct noise comprehension. However, several studies performed in this field concern the flame acoustic radiation towards the free far-field, whereas in the case of confined systems like aero-engines another source of noise appears to be relevant: the entropy noise. Indeed, following the work of Tsien ${ }^{11}$ and Crocco ${ }^{12}$ about nozzles, Candel concludes in his thesis ${ }^{13}$ that entropy spots, inherent to the unsteady combustion process, can represent an important source of noise when considering gas expansion through the engine turbine stages. Candel considers a relative temperature fluctuation of $5 \%$, and calculates a corresponding sound pressure level at the nozzle exhaust of about 120 dB. Marble \& Candel ${ }^{14,15}$ obtain solutions for planar waves within compact nozzles, and within finite length nozzles with assumed linear axial-velocity evolution. In the same period Ffowcs Williams \& Howe ${ }^{16}$ propose an extended theory for general entropy spots in low Mach number nozzle flows. Stow \& Dowling ${ }^{17}$ show that for an annular duct with a nozzle, the relations found by Marble \& Candel for compact nozzles apply to first-order even when circumferential modes are present. The compact interactions idea of Marble \& Candel was extended to cylindrical 2-D flows by Cumpsty \& Marble ${ }^{18}$ and applied on commercial aero-engines. ${ }^{19}$ Considering that the principal noise mechanism is the indirect one, Cumpsty \& Marble presume the relative temperature fluctuation amplitude and spectrum, and obtain quite good results for low jet velocities: the so-called "excess-noise". But Cumpsty \& Marble agree to say that although the agreement of the measurements and predictions strongly supports that the indirect noise mechanism is the major core-noise generation process, this conclusion is not definitive until a clear separation of the different effects is performed. Starting from the heat release fluctuation, they provide the main ideas to do this analytically and briefly comment the expected results. The aim of the present article is precisely to develop this work, but in a purely one-dimensional case. It should be mentioned that Muthukrishnan et $a l .{ }^{20}$ experimentally investigate the core-noise sources separation and also conclude that for choked nozzles, entropy noise seems to be the main core-noise source. More recently Bake et $a .^{21-23}$ worked on the subject, but the separation of direct and indirect combustion noise in a real-case is still difficult to evaluate. On one hand, entropy fluctuations are controlled by complicated aerodynamical, thermal and chemical phenomena. Turbulent mixing and diffusion can also strongly affect entropy waves amplitudes at the nozzle inlet, generally speaking. On the other hand, the acoustic cavity modes of the chamber can also significantly change the acoustic levels. ${ }^{24,25}$ In the present study, only the most significant parameters are considered in order to establish simple analytical scaling laws for direct and indirect noise in aero-engines. To compare direct and indirect combustion noise, entropy and acoustic waves will be assumed to be directly linked to heat release fluctuations, and a very simple case of 
generic combustor is considered: a combustion chamber followed by a nozzle (Fig. 2). The

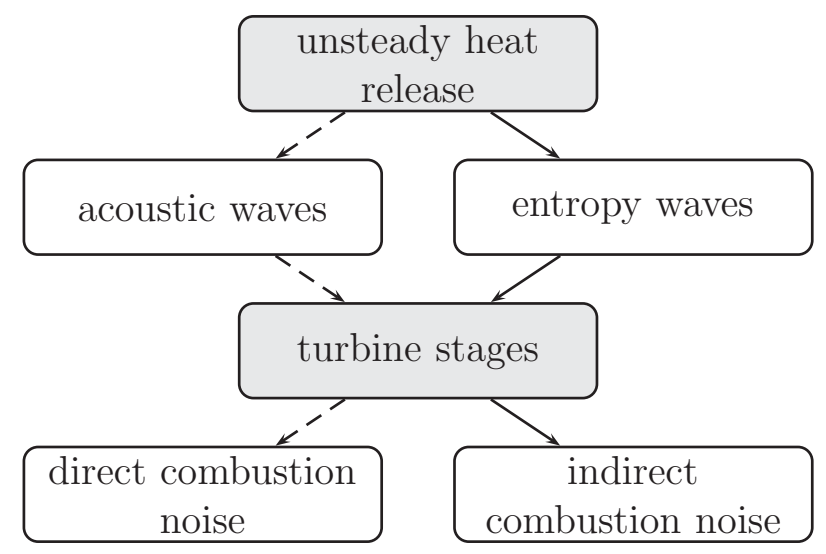

Figure 1. The two main mechanisms for noise generation from confined flames: direct $(---)$ and indirect $(-)$ noise.

combustion chamber and the turbine stages will be represented by a quasi-1-D system. The combustion zone will be modeled by an infinitely thin heat release fluctuation in a constant section duct (generating acoustic and entropy waves) connected to a quasi-1-D nozzle representing the turbine stages (for the transmission and the generation of acoustic waves). These

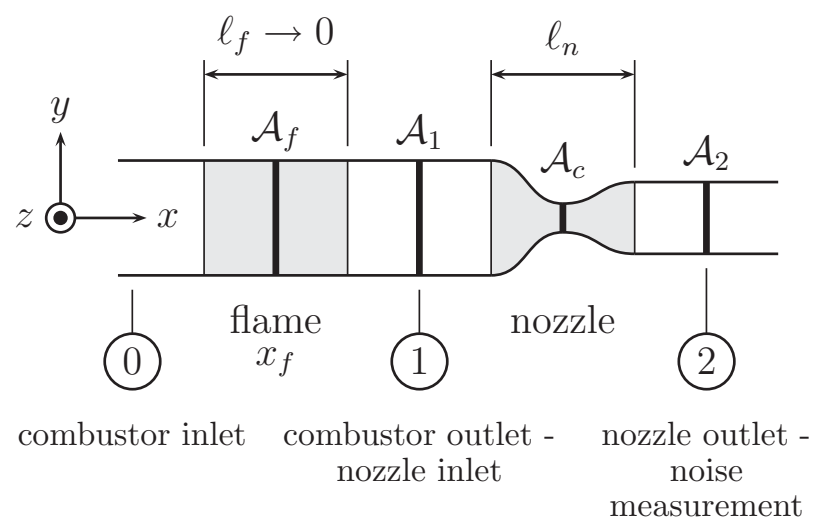

Figure 2. A generic model to evaluate direct and indirect combustion noise.

two elements are handled individually as shown in Fig. 3, and the feedback on the flame, of the acoustic waves traveling upward is not taken into account. Thus, the separation between direct and indirect noise is simple to perform since the global system is assumed to be linear. The combustion chamber creates the acoustic and entropy waves feeding the nozzle. The nozzle then generates the out-going direct or indirect noise depending on the nature of the in-going waves (acoustic or entropy respectively). The waves generated by the combus- 
tion zone are calculated analytically, considering an isolated heat release fluctuation. This model provides explicitly acoustic and entropy waves for the second part of the calculations: the transmission-generation of acoustic waves through the nozzle. The calculation of the transmission of acoustic waves through the nozzle (direct mechanism), and of the generation of acoustic waves from entropy waves within the nozzle (indirect mechanism) is performed both analytically using the results of Marble \& Candel $^{15}$ for compact nozzles, and numerically by solving the Euler equations in the time-domain for a 2-D nozzle with a quasi-1-D behavior. The present method thus leads to two main approaches for the calculation of the indirect-to-direct noise ratio: a fully-analytical method and a semi-analytical method. In both cases, the calculations of the waves due to combustion and calculations of the waves transmission-generation through the nozzle are independent.
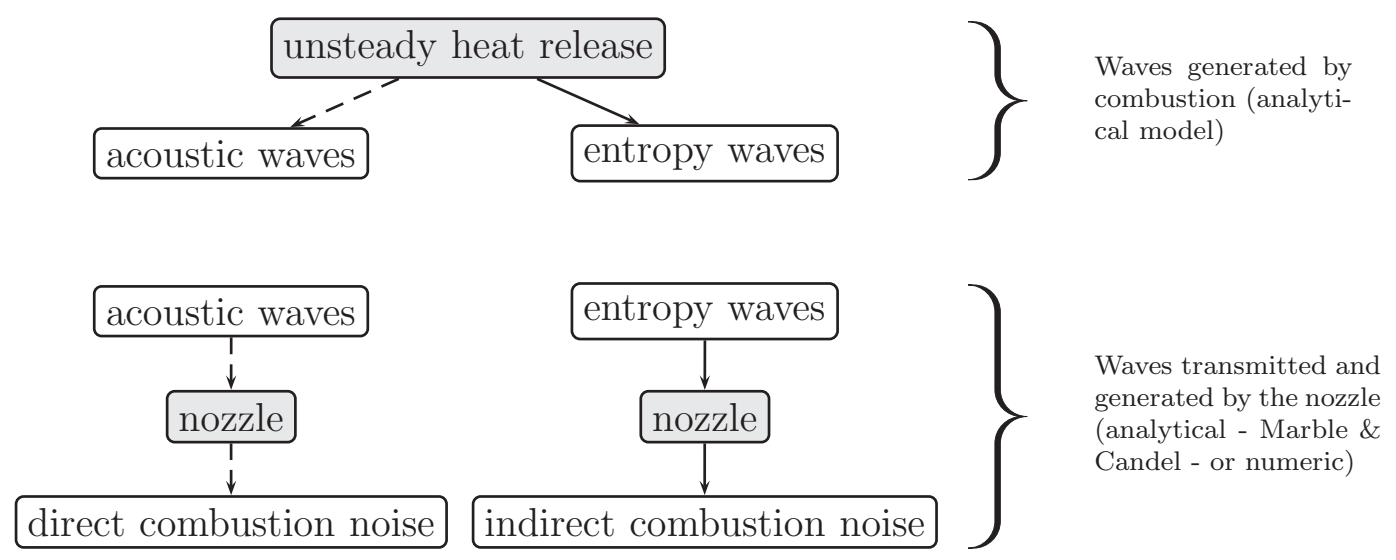

Figure 3. Strategy for the calculation of the ratio between direct and indirect noise.

Analytical calculations of acoustic and entropy waves generated by combustion will be first reviewed in Sec. II. Acoustic waves transmission and generation by the nozzle, obtained analytically and numerically, are then presented in Sec. III. Finally, the ratio between direct and indirect noise is presented in Sec. IV.

\section{Acoustic and entropy waves generation in the combustion chamber}

A subsonic flow is assumed in the combustion region. Viscous and 3-D effects are neglected and a one-dimensional heat release region is assumed to represent the combustion zone. The flow is defined with the mass density $\rho$, the velocity $u$ and the heat release $\dot{Q}(t)$. The flame length $\ell_{f}$ is assumed to be small compared with the acoustic and entropy 
wavelengths, so that the heat release per unit volume $\dot{q}$ can be expressed as:

$$
\dot{q}(x, y, z, t)=\delta\left(x-x_{f}\right) \dot{Q}(t) / \mathcal{A}_{f}
$$

where $\delta$ is the Dirac distribution and $\mathcal{A}_{f}$ the cross-section area of the combustion chamber. The heat release $\dot{Q}(t)$ results from the following space integration of $\dot{q}(x, y, z, t)$ :

$$
\dot{Q}(t)=\iiint_{-\infty}^{+\infty} \dot{q}(x, y, z, t) d x d y d z
$$

The steady heat release of the flame model is considered negligible (cold flame) so that the mean flow is assumed isentropic. The cold flame assumption has been used by many authors to obtain analytical scaling regarding thermoacoustic instabilities, ${ }^{26}$ but it can have a nonnegligible effect as shown by Dowling ${ }^{27}$ which is ignored here. The flow is caracterised by the mass flow $\dot{m}$, the total enthalpy $h_{t}$ and the entropy $s$. The mass flow $\dot{m}$ and the entropy $s$ can be written as follows:

$$
\begin{aligned}
\dot{m} & =\rho u \mathcal{A} \\
s & =c_{v} \ln \left(\frac{p}{\rho^{\gamma}}\right)
\end{aligned}
$$

or for small temporal perturbations:

$$
\begin{aligned}
\frac{\dot{m}^{\prime}}{\overline{\dot{m}}} & =\frac{1}{\overline{\mathcal{M}}} \frac{u^{\prime}}{\bar{c}}+\frac{p^{\prime}}{\gamma \bar{p}}-\frac{s^{\prime}}{c_{p}} \text { and } \\
\frac{s^{\prime}}{c_{p}} & =\frac{p^{\prime}}{\gamma \bar{p}}-\frac{\rho^{\prime}}{\bar{\rho}}
\end{aligned}
$$

Heat capacities and the composition of the gas are assumed to be constant, so that the total enthalpy is defined by $h_{t}=c_{p} T_{t}$, where $T_{t}$ is the total temperature:

$$
T_{t}=T\left(1+\frac{\gamma-1}{2} \mathcal{M}^{2}\right)
$$

The fluctuation of the Mach number $\mathcal{M}$ is:

$$
\frac{\mathcal{M}^{\prime}}{\overline{\mathcal{M}}}=\frac{1}{\overline{\mathcal{M}}} \frac{u^{\prime}}{\bar{c}}-\frac{\gamma-1}{2} \frac{p^{\prime}}{\gamma \bar{p}}-\frac{1}{2} \frac{s^{\prime}}{c_{p}},
$$

The fluctuations of total temperature as a function of velocity, pressure and entropy 
perturbations can be written using Eq. $(6,8)$ and the state equation for small perturbations:

$$
\frac{T_{t}^{\prime}}{\bar{T}_{t}}=\frac{1}{1+\frac{\gamma-1}{2} \overline{\mathcal{M}}^{2}}\left((\gamma-1) \overline{\mathcal{M}} \frac{u^{\prime}}{\bar{c}}+(\gamma-1) \frac{p^{\prime}}{\gamma \bar{p}}+\frac{s^{\prime}}{c_{p}}\right)
$$

To scale direct and indirect combustion noise, the dimensionless acoustic $\left(w^{+}\right.$and $\left.w^{-}\right)$ and entropy $\left(w^{\mathrm{S}}\right)$ waves created by the compact flame of the combustion chamber must be assessed. These waves are defined as follows:

$$
\begin{aligned}
w^{+} & =\frac{p^{\prime}}{\gamma \bar{p}}+\frac{u^{\prime}}{\bar{c}} \\
w^{-} & =\frac{p^{\prime}}{\gamma \bar{p}}-\frac{u^{\prime}}{\bar{c}} \\
w^{\mathrm{S}} & =\frac{p^{\prime}}{\gamma \bar{p}}-\frac{\rho^{\prime}}{\bar{\rho}}
\end{aligned}
$$

The wave $w^{+}$is related to the propagation speed $u+c$, while the wave $w^{-}$is related to the propagation speed $u-c$. The wave $w^{\mathrm{S}}$ propagates at the convective speed $u$ and transports entropy.

Considering the heat release $\dot{Q}$ through the heating region according to Fig. 4, the balance equations of mass flow, total enthalpy and entropy at the flame front yield:

$$
\begin{aligned}
(\dot{m})_{0} & =(\dot{m})_{1} \\
\left(\dot{m} h_{t}\right)_{0}+\dot{Q} & =\left(\dot{m} h_{t}\right)_{1} \\
(\dot{m} s)_{0}+\frac{\dot{Q}}{T} & =(\dot{m} s)_{1}
\end{aligned}
$$

where the subscripts (0) and (1) correspond respectively to the quantities upstream and downstream from the flame. The mean heat release is zero, so that the mean temperature $\bar{T}$, Mach number $\overline{\mathcal{M}}$, total temperature $\bar{T}_{t}$ and entropy $\bar{s}$ do not change through the flame model. For small temporal perturbations and using mass flow balance Eq. (13), the entropy balance Eq. (15) leads to:

$$
\left(\frac{s^{\prime}}{c_{p}}\right)_{0}+\frac{\dot{Q}^{\prime}}{\dot{\bar{m}} c_{p} \bar{T}}=\left(\frac{s^{\prime}}{c_{p}}\right)_{1}
$$

Equation (14) can be modified in the same way, and using the expression of the fluctuations of the total temperature Eq. (9), the total enthalpy balance Eq. (14) for small temporal 
perturbations leads to:

$$
\begin{aligned}
(\gamma-1) \overline{\mathcal{M}}\left(\frac{u^{\prime}}{\bar{c}}\right)_{0}+(\gamma-1) & \left(\frac{p^{\prime}}{\gamma \bar{p}}\right)_{0}+\left(\frac{s^{\prime}}{c_{p}}\right)_{0}+\frac{\dot{Q}^{\prime}}{\overline{\dot{m}} c_{p} \bar{T}}= \\
& (\gamma-1) \overline{\mathcal{M}}\left(\frac{u^{\prime}}{\bar{c}}\right)_{1}+(\gamma-1)\left(\frac{p^{\prime}}{\gamma \bar{p}}\right)_{1}+\left(\frac{s^{\prime}}{c_{p}}\right)_{1}
\end{aligned}
$$

The heat release is supposed to be known, and the three in-going waves have to be imposed in order to obtain the out-going waves $w_{1}^{+}, w_{1}^{\mathrm{S}}$ and $w_{0}^{-}$using the three balance equations Eq. $(13,14,15)$ as a function of the heat release. Except for the heat release, the combustor

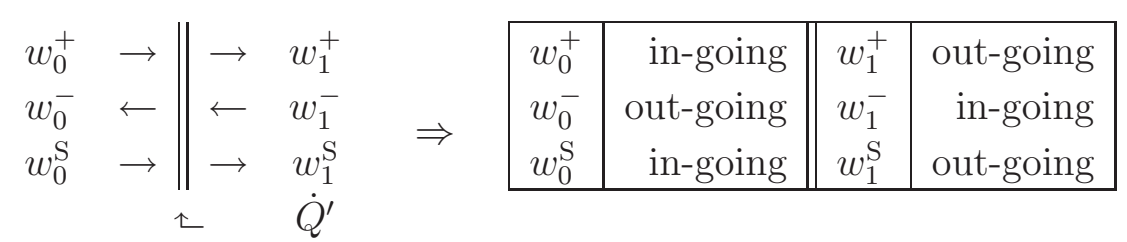

Table 1. In-going and out-going waves for the compact flame.

is assumed to be isolated, that is to say that the in-going acoustic waves $w_{0}^{+}$and $w_{1}^{-}$are equal to zero, as well as the in-going entropy wave $w_{0}^{\mathrm{S}}$. Considering the last assumption $\left(w_{0}^{\mathrm{S}}=0\right)$ and using Eq. (16), the out-going entropy $w_{1}^{\mathrm{S}}$ can be expressed as:

$$
w_{1}^{\mathrm{S}}=\frac{\dot{Q}^{\prime}}{\overline{\dot{m}} c_{p} \bar{T}}
$$

The fluctuation of the mass flow Eq. (5) can be written upstream (0) and downstream (1) from the flame and expressed as a function of waves, instead of the fluctuations of velocity, pressure and entropy. Then, Eq. (17) related to the fluctuations of total enthalpy can also be written as a function of waves. Using Eq. (18) and the assumption that the flame is isolated $\left(w_{0}^{+}=0, w_{1}^{-}=0\right.$ and $\left.w_{0}^{\mathrm{S}}=0\right)$ one can show that the expression of the out-going acoustic wave $w_{1}^{+}$generated by the heat release fluctuation is:

$$
w_{1}^{+}=\frac{\overline{\mathcal{M}}}{1+\overline{\mathcal{M}}} \frac{\dot{Q}^{\prime}}{\overline{\dot{m}} c_{p} \bar{T}}
$$

Finally, Eq. $(18,19)$ lead to the ratio between the acoustic wave $w_{1}^{+}$and the entropy wave $w_{1}^{\mathrm{S}}$ generated by the combustion zone and propagating downstream the combustion chamber:

$$
\frac{w_{1}^{+}}{w_{1}^{\mathrm{S}}}[\mathrm{CC}]=\frac{\overline{\mathcal{M}}}{1+\overline{\mathcal{M}}}
$$

where [CC] refers to waves produced in the combustion chamber. Equation (20) shows that, for a compact flame, the ratio between acoustic and entropy waves depends only on the Mach 


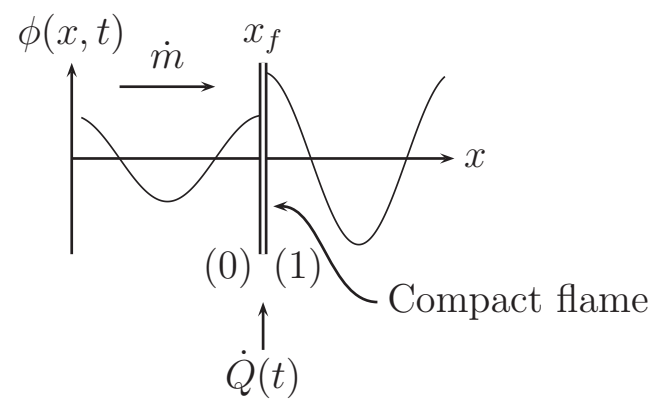

Figure 4. Compact flame illustration regarding a quantity $\phi$ upstream (0) and downstream (1) from the heating region.

number in the flame zone $\overline{\mathcal{M}}$ and no more on the heat release fluctuations. This result allows studying the ratio between indirect and direct combustion noise mechanisms independently of the exact nature of heat release fluctuations, which are the sources of each mechanism.

\section{Waves transmission and generation through a nozzle}

The transmission and the generation of acoustic and entropy waves through the nozzle are obtained using two different methods. The first one is based on the analytical development of Marble and Candel $^{15}$ assuming that the nozzle is compact (frequency is low). In their work, the authors assume a quasi-1-D nozzle flow and quasi-steady perturbations of mass flow, energy and entropy, leading to relations between the different waves that depend on the inlet and outlet Mach numbers. This approach is similar to the one used for the compact flame of Sec. II, and the relations between waves for the nozzle are reviewed in the first part of this section. The second method to obtain the acoustic response of the nozzle is based on a numerical simulation of the quasi-1-D nozzle flow, by solving the Euler equations. ${ }^{28}$ This second method is valid for all frequencies as long as the waves remain one-dimensional, and will be used here to evaluate the compact nozzle assumption of the analytical approach in the low frequency limit. The numerical approach is presented in the second part of this section.

\section{A. Analytical approach}

Following the analysis of Marble and Candel, the flow is supposed to be one-dimensional. Similarly to Sec. II, $u$ stands for the axial velocity, $\rho$ for the mass density and $\mathcal{A}$ for the nozzle cross-section area. The mass flow $\dot{m}$ and the entropy $s$ are defined by Eq. $(3,4)$. Assuming that the nozzle is isolated, the total enthalpy is conserved and thus the total temperature too. Note that the total temperature $T_{t}$ is in this case always conserved, even 
for a non-isentropic mean flow in the nozzle (that is to say through a shock for instance). The total temperature is defined by Eq. (7) and the expression of the fluctuations of total temperature entering the nozzle as a function of speed, pressure and entropy perturbations is the same as in Sec. II - Eq. (9). Under the assumption of compact nozzle (long wavelengths compared with the nozzle length $\ell_{n}$ - see Fig. 5), there is no delay or distortion between the inlet and the outlet of the nozzle. As a result, mass flow, total temperature and entropy are conserved through the nozzle. The quantities upstream and downstream from the nozzle are respectively subscripted (1) and (2). The conservation equations Eq. (5, 6, 9) can be

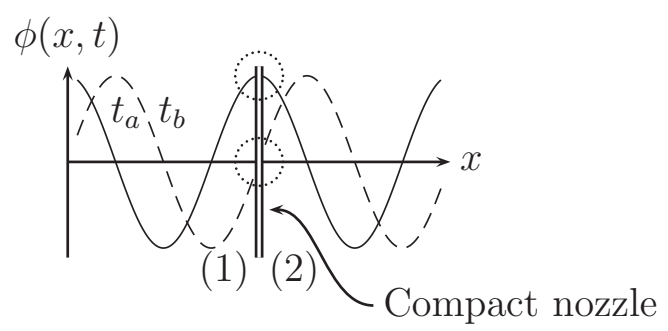

Figure 5. Compact nozzle illustration: The quantity $\phi$, conserved throughout the nozzle, is the same upstream (1) and downstream (2) at each instant.

written as a system of equations, function only of upstream and downstream Mach numbers and waves (acoustic and entropy). This system is composed of three equations involving six waves, thus the in-going waves have to be imposed. In the case of an unchoked nozzle, the flow is totally subsonic and the $w_{2}^{-}$wave is in-going, so that waves $w_{1}^{+}, w_{1}^{\mathrm{S}}$ et $w_{2}^{-}$can be imposed. Finally, the system is composed of three equations and three unknown waves. Since the system is linear, mechanisms can be separated by setting $w_{1}^{+} \neq 0, w_{1}^{\mathrm{S}}=0$ and

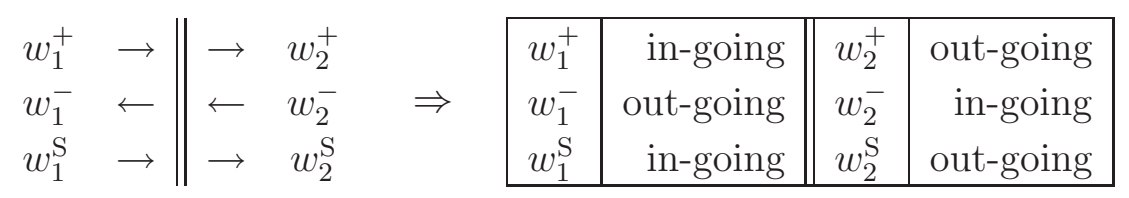

Table 2. In-going and out-going waves for the unchoked nozzle case.

$w_{2}^{-}=0$ in a first step to study only the "acoustic" response of the nozzle to an "acoustic" perturbation. This case is called [AA]. It is also possible to set $w_{1}^{+}=0, w_{1}^{\mathrm{S}} \neq 0$ and $w_{2}^{-}=0$ in order to study the "acoustic" response of the nozzle to an "entropy" perturbation, and this case is called $[\mathrm{SA}]$. Using Eq. $(5,6,9)$ at the inlet and the outlet of the nozzle for the 
cases $[\mathrm{AA}]$ and $[\mathrm{SA}]$ gives:

$$
\begin{aligned}
& \frac{w_{2}^{+}}{w_{1}^{+}}[\mathrm{AA}]=\left(\frac{2 \overline{\mathcal{M}}_{2}}{1+\overline{\mathcal{M}}_{2}}\right)\left(\frac{1+\overline{\mathcal{M}}_{1}}{\overline{\mathcal{M}}_{1}+\overline{\mathcal{M}}_{2}}\right)\left(\frac{1+\frac{1}{2}(\gamma-1) \overline{\mathcal{M}}_{2}^{2}}{1+\frac{1}{2}(\gamma-1) \overline{\mathcal{M}}_{1} \overline{\mathcal{M}}_{2}}\right) \\
& \frac{w_{2}^{+}}{w_{1}^{\mathrm{S}}}[\mathrm{SA}]=\left(\frac{\frac{1}{2} \overline{\mathcal{M}}_{2}-\overline{\mathcal{M}}_{1}}{1+\overline{\mathcal{M}}_{2}}\right)\left(\frac{1}{1+\frac{1}{2}(\gamma-1) \overline{\mathcal{M}}_{1} \overline{\mathcal{M}}_{2}}\right)
\end{aligned}
$$

In the case of an isentropic choked nozzle, the flow is subsonic in the convergent part of the nozzle and totally supersonic in the divergent part and wave $w_{2}^{-}$cannot be imposed, since it is out-going. In the case of an isentropic choked nozzle, only two waves can be imposed so

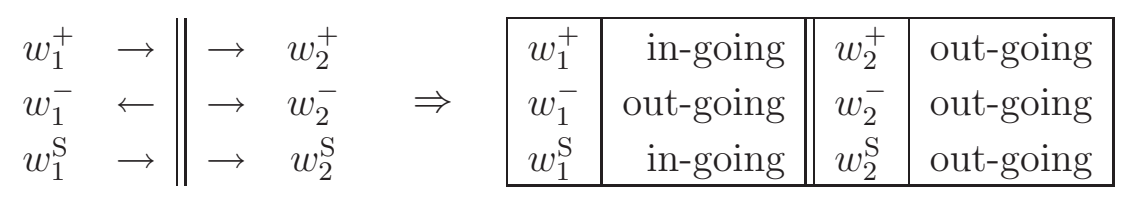

Table 3. In-going and out-going waves for the choked nozzle case.

that the system is composed of four unknown waves for three equations only. The missing equation is obtained by stating that the flow at the nozzle throat is sonic so that the relation between the Mach number $\mathcal{M}$ and the cross-section area ratio can be written as follows:

$$
\frac{\mathcal{A}}{\mathcal{A}_{c}}=\frac{1}{\mathcal{M}}\left[\frac{2}{\gamma+1}\left(1+\frac{\gamma-1}{2} \mathcal{M}^{2}\right)\right]^{\frac{1}{2} \frac{\gamma+1}{\gamma-1}}
$$

where $\mathcal{A}_{c}$ is the cross-section area at the nozzle throat. One can easily show from Eq. (23) that for the choked nozzle case, the temporal fluctuation of the Mach number $\mathcal{M}^{\prime} / \overline{\mathcal{M}}$ is zero. Then, using the expression of the fluctuation of the Mach Eq. (8):

$$
\frac{1}{\overline{\mathcal{M}}} \frac{u^{\prime}}{\bar{c}}-\frac{\gamma-1}{2} \frac{p^{\prime}}{\gamma \bar{p}}-\frac{1}{2} \frac{s^{\prime}}{c_{p}}=0
$$

The entropy $s$ and the total temperature $T_{t}$ are constant through the nozzle, so that the total pressure $p_{t}$ is also. The total pressure can be expressed as follows:

$$
p_{t}=p\left(1+\frac{\gamma-1}{2} \mathcal{M}^{2}\right)^{\frac{\gamma}{\gamma-1}}
$$

The temporal fluctuation of total pressure of Eq. (25) can be written:

$$
\frac{p_{t}^{\prime}}{\bar{p}_{t}}=\frac{p^{\prime}}{\bar{p}}+\frac{\gamma \overline{\mathcal{M}}^{2}}{1+\frac{\gamma-1}{2} \overline{\mathcal{M}}^{2}} \frac{\mathcal{M}^{\prime}}{\overline{\mathcal{M}}}
$$


and shows that the fluctuations $p^{\prime} /(\gamma \bar{p})$ are the same on both sides of the nozzle since the Mach number fluctuation is zero:

$$
\left(\frac{p^{\prime}}{\gamma \bar{p}}\right)_{1}=\left(\frac{p^{\prime}}{\gamma \bar{p}}\right)_{2}
$$

Finally, Eq. $(6,24,27)$ used at the inlet and the outlet of the nozzle, yield:

$$
\begin{aligned}
& \frac{w_{2}^{+}}{w_{1}^{+}}[\mathrm{AA}]=\frac{1+\frac{1}{2}(\gamma-1) \overline{\mathcal{M}}_{2}}{1+\frac{1}{2}(\gamma-1) \overline{\mathcal{M}}_{1}} \\
& \frac{w_{2}^{+}}{w_{1}^{\mathrm{S}}}[\mathrm{SA}]=\frac{\frac{1}{2}\left(\overline{\mathcal{M}}_{2}-\overline{\mathcal{M}}_{1}\right)}{1+\frac{1}{2}(\gamma-1) \overline{\mathcal{M}}_{1}}
\end{aligned}
$$

The set of equations Eq. $(21,22,28,29)$ provides analytical expressions for the out-going waves $w_{2}^{+}$as a function of the in-going ones $\left(w_{1}^{+}\right.$and $\left.w_{1}^{S}\right)$ and the mean inlet $\left(\overline{\mathcal{M}}_{1}\right)$ and outlet $\left(\overline{\mathcal{M}}_{2}\right)$ Mach numbers for the compact nozzle.

\section{B. Numerical approach}

The previous analytical relations are based on the nozzle compactness assumption and are valid only for the low frequency limit. In order to validate this assumption and extend the model for the nozzle to a larger range of frequencies, an unsteady simulation based on Euler equations of the flow within the nozzle has been performed. Acoustic and entropy perturbations are generated at the inlet of the computational domain in a simple one-dimensional isentropic nozzle flow, and out-going noise is directly measured in the simulation.

\section{Numerical method}

The numerical tool used to solve the flow within the nozzle is the AVBP ${ }^{29,30}$ code. AVBP is a finite-volume cell-vertex code which can solve 3-D compressible Navier-Stokes equations on unstructured meshes, but it is used here on a 2-D regular mesh without viscous terms. The mesh is two-dimensional ( $476 \times 5$ with 320 nodes in the nozzle - Fig. 6$)$, and the evolution of the transversal coordinate is small enough to assume that the flow in the nozzle is quasi-onedimensional. There is about 320 nodes in the axial direction of the nozzle. The numerical

Figure 6. Mesh of the nozzle corresponding to the case with $\mathcal{M}_{1}=0.050$ and $\mathcal{M}_{2}=1.600$.

computations have been performed with the Lax-Wendroff scheme which is second-order in space and time accurate, with a Courant-Friedrichs-Lewy number of 0.5. Preliminary tests 
performed with the same solver on acoustic and entropy waves propagation were used to verify that the results were independent of the mesh and that dispersion and dissipation errors were very small.

\section{Nozzle geometry and flow parameters}

Since an inviscid, one-dimensional and compressible flow is also considered in the simulation, the mean Mach numbers $\overline{\mathcal{M}}_{1}$ and $\overline{\mathcal{M}}_{2}$ only depend on the cross-section area ratio $\mathcal{A}_{1} / \mathcal{A}_{2}$ for the unchoked nozzle case, and of the cross-section area ratios $\mathcal{A}_{1} / \mathcal{A}_{c}$ and $\mathcal{A}_{2} / \mathcal{A}_{c}$ for the isentropic choked nozzle case - Eq. (23). For the choked nozzle case, the nozzle is convergent and divergent, whereas it is simply convergent for the unchoked nozzle case. To calculate the section area ratio $\mathcal{A}_{1} / \mathcal{A}_{2}$ for the unchoked case, the following relation is used:

$$
\frac{\mathcal{A}_{1}}{\mathcal{A}_{2}}=\frac{\overline{\mathcal{M}}_{2}}{\overline{\mathcal{M}}_{1}}\left[\frac{1+\frac{\gamma-1}{2} \overline{\mathcal{M}}_{1}^{2}}{1+\frac{\gamma-1}{2} \overline{\mathcal{M}}_{2}^{2}}\right]^{\frac{1}{2} \frac{\gamma+1}{\gamma-1}}
$$

The different values of cross-section area ratios used in the present work are presented in Tab. 4 as a function of inlet Mach number $\overline{\mathcal{M}}_{1}$ and outlet Mach number $\overline{\mathcal{M}}_{2}$ for a specific heat capacities ratio $\gamma$ of 1.32 . The static pressure and temperature are imposed at the inlet

\begin{tabular}{|c|c|c|c|c|}
\hline Case & \multicolumn{2}{|c|}{ Unchoked } & \multicolumn{2}{c|}{ Choked } \\
\hline \hline$\overline{\mathcal{M}}_{1}$ & 0.400 & 0.800 & 1.200 & 1.600 \\
\hline \multirow{2}{*}{0.025} & $\mathrm{~A} 1 / \mathrm{Ac}=14.604$ & $\mathrm{~A} 1 / \mathrm{Ac}=22.482$ & $\mathrm{~A} 1 / \mathrm{Ac}=23.365$ & $\mathrm{~A} 1 / \mathrm{Ac}=23.365$ \\
& $\mathrm{~A} 2 / \mathrm{Ac}=1.000$ & $\mathrm{~A} 2 / \mathrm{Ac}=1.000$ & $\mathrm{~A} 2 / \mathrm{Ac}=1.032$ & $\mathrm{~A} 2 / \mathrm{Ac}=1.267$ \\
\hline 0.050 & $\mathrm{~A} 1 / \mathrm{Ac}=7.310$ & $\mathrm{~A} 1 / \mathrm{Ac}=11.253$ & $\mathrm{~A} 1 / \mathrm{Ac}=11.695$ & $\mathrm{~A} 1 / \mathrm{Ac}=11.695$ \\
& $\mathrm{~A} 2 / \mathrm{Ac}=1.000$ & $\mathrm{~A} 2 / \mathrm{Ac}=1.000$ & $\mathrm{~A} 2 / \mathrm{Ac}=1.032$ & $\mathrm{~A} 2 / \mathrm{Ac}=1.267$ \\
\hline 0.100 & $\mathrm{~A} 1 / \mathrm{Ac}=3.671$ & $\mathrm{~A} 1 / \mathrm{Ac}=5.651$ & $\mathrm{~A} 1 / \mathrm{Ac}=5.873$ & $\mathrm{~A} 1 / \mathrm{Ac}=5.873$ \\
& $\mathrm{~A} 2 / \mathrm{Ac}=1.000$ & $\mathrm{~A} 2 / \mathrm{Ac}=1.000$ & $\mathrm{~A} 2 / \mathrm{Ac}=1.032$ & $\mathrm{~A} 2 / \mathrm{Ac}=1.267$ \\
\hline
\end{tabular}

Table 4. Geometric cross-section area-ratio values for the different Mach number cases.

of the nozzle $\left(p_{1}=800.0 \mathrm{kPa}, T_{1}=1300 \mathrm{~K}\right)$, and are the same for all cases. The static pressure $p_{2}$ is also imposed at the outlet and is chosen to obtain an isentropic flow and thus the correct target Mach numbers.

\section{Numerical boundary conditions and computations}

Like for the analytical approach, the acoustic response of the nozzle at the outlet is computed for a case where the in-going waves imposed at the nozzle inlet are entropy (case [SA]), and for a case where these waves are acoustic (case $[\mathrm{AA}]$ ). In this numerical computation, totally 
non-reflecting boundary conditions are imposed, $\left(w_{1}^{+}\right.$independent of $w_{1}^{-}$at the nozzle inlet, and $w_{2}^{-}=0$ for the subsonic nozzle outlet case), and the desired in-going perturbation added. ${ }^{30,31}$ The in-going waves at the nozzle inlet are imposed as follows:

$$
\left\{\begin{array}{l}
w_{1}^{\mathrm{S}}(t)=n(t) \\
w_{1}^{+}(t)=0
\end{array} \text { for the case }[\mathrm{SA}]\right.
$$

and

$$
\left\{\begin{array}{l}
w_{1}^{\mathrm{S}}(t)=0 \\
w_{1}^{+}(t)=n(t)
\end{array} \text { for the case }[\mathrm{AA}]\right.
$$

where $n(t)$ is a filtered white noise signal. The perturbations $n(t)$ are small enough to neglect non-linear effects. As a result, one single computation with a filtered white noise signal imposed at the inlet can be performed to obtain the acoustic response of the nozzle to a large range of frequencies. The cut-off frequency of the filtered white noise signal and the size of the biggest cell are chosen in order to solve the smallest wavelength over 20 nodes in the most unfavorable case, that is to say the case of entropy waves forcing at low Mach numbers. The smallest acoustic wave length taken into account here is of the order of half the nozzle length (this corresponds to about 1/16 of the nozzle length for the entropy wave in the most unfavorable case). However, to avoid border-effects in the frequency-domain due to the low-pass filter, and to have a better numerical precision in the range of interest, the results presented in the next section are given for a reduced frequency range. The upper frequency limit of the presented results corresponds to a grid resolution of 50 nodes for the smallest entropy wavelength.

Temporal evolutions of the different waves are recorded at the nozzle inlet and outlet, and the Welch's method ${ }^{32}$ is used to compute the spectral power density of the waves' signals and thus establish the desired spectral transfer functions. A first computation (case [SA]) provides the transfer function

$$
\sqrt{\mathrm{PW}\left\{w_{2}^{+}\right\} / \mathrm{PW}\left\{w_{1}^{\mathrm{S}}\right\}}
$$

relevant of the indirect mechanism, whereas a second computation (case [AA]) provides the transfer function

$$
\sqrt{\mathrm{PW}\left\{w_{2}^{+}\right\} / \mathrm{PW}\left\{w_{1}^{+}\right\}}
$$

relevant of the direct mechanism. These numerical transfer functions of the nozzle, in combination with the analytical results for waves generated by the combustion zone, are then used to calculate the semi-analytic indirect-to-direct ratio. 


\section{Results}

Results of Sec. II and Sec. III are used to calculate the ratio $\eta$ between amplitude $w_{2, \text { ind }}^{+}$of the acoustic wave generated indirectly and the amplitude $w_{2, \text { dir }}^{+}$of the acoustic wave generated directly as described in Tab. 5 . The acoustic wave $w_{2 \text {,ind }}^{+}$generated by the indirect mechanism

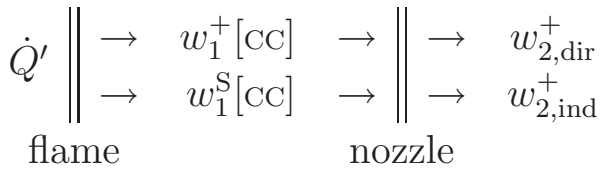

Table 5. Definition of the direct and indirect acoustic waves.

is expressed using the entropy wave $w_{1}^{\mathrm{S}}[\mathrm{CC}]$ produced by the flame, and the transfer function of the nozzle of the case [SA]. The acoustic wave $w_{2, \text { dir }}^{+}$generated by the direct mechanism is however expressed using the acoustic wave $w_{1}^{+}[\mathrm{CC}]$ produced by the flame, and the transfer function of the nozzle of the case $[\mathrm{AA}]$. The ratio $\eta$ is defined as follows:

$$
\eta=\frac{w_{2, \text { ind }}^{+}}{w_{2, \text { dir }}^{+}}
$$

where:

$$
w_{2, \mathrm{dir}}^{+}=\frac{w_{2}^{+}}{w_{1}^{+}}[\mathrm{AA}] \cdot w_{1}^{+}[\mathrm{CC}] \quad \text { and } \quad w_{2, \text { ind }}^{+}=\frac{w_{2}^{+}}{w_{1}^{\mathrm{S}}}[\mathrm{SA}] \cdot w_{1}^{\mathrm{S}}[\mathrm{CC}]
$$

The ratio $\eta$ can be calculated, as mentioned previously, either in a fully-analytic way (using the analytical relations of Marble \& Candel for the transmission and generation of waves through a compact nozzle, and the analytic relation for waves generated by combustion Sec. III-A), or in a semi-analytic manner (using the numerical calculations for the transmission and generation of waves through 1-D nozzle flow, and the same analytical relation for waves generated by combustion - Sec. III-B):

$$
\eta=\underbrace{\frac{w_{2}^{+}}{w_{1}^{\mathrm{S}}}[\mathrm{SA}]}_{\text {analytic or numeric }} \underbrace{\frac{w_{1}^{\mathrm{S}}}{w_{1}^{+}}[\mathrm{CC}]}_{\text {analytic }} \underbrace{\frac{w_{1}^{+}}{w_{2}^{+}}[\mathrm{AA}]}_{\text {analytic or numeric }}
$$

The fully-analytic expression of the ratio $\eta$ is established using Eq. (20) giving the ratio between acoustic and entropy waves produced by the combustion chamber (case [CC]), and Eq. $(21,22,28,29)$ giving the transmitted and generated waves by a compact nozzle. For 
this approach, the expression of $\eta$ is:

$$
\begin{aligned}
& \eta=\frac{1}{\overline{\mathcal{M}}_{1}} \frac{\left(\overline{\mathcal{M}}_{2}-\overline{\mathcal{M}}_{1}\right)\left(\overline{\mathcal{M}}_{2}+\overline{\mathcal{M}}_{1}\right)}{2\left(1+\frac{1}{2}(\gamma-1) \overline{\mathcal{M}}_{2}^{2}\right)} \quad \text { for the unchoked nozzle } \\
& \eta=\frac{1+\overline{\mathcal{M}}_{1}}{\overline{\mathcal{M}}_{1}} \frac{\overline{\mathcal{M}}_{2}-\overline{\mathcal{M}}_{1}}{2\left(1+\frac{1}{2}(\gamma-1) \overline{\mathcal{M}}_{2}\right)} \quad \text { for the choked nozzle }
\end{aligned}
$$

The ratios between indirect and direct noise $\eta$, calculated with the fully-analytic and semianalytic approaches, are plotted hereafter for the Mach numbers defined previously, as a function of reduced pulsation $\Omega=\omega \ell_{n} / \bar{c}_{1}$ related to the pulsation $\omega$, the speed of sound at the nozzle inlet $\bar{c}_{1}$ and the nozzle length $\ell_{n}$. Figure 7 shows that for this simple case,

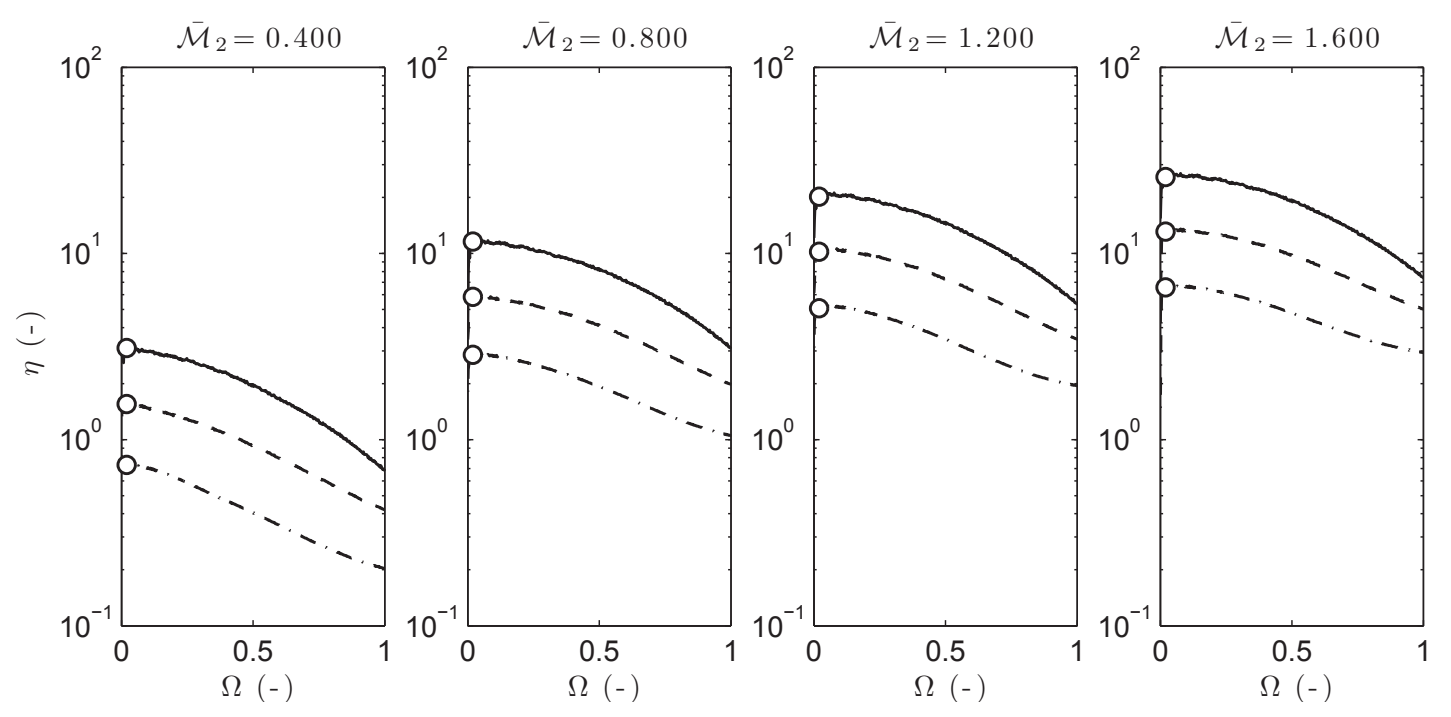

Figure 7. Estimation of the ratio $\eta$ between indirect and direct noise. Chain dotted line $\cdot-\cdot-$. Mach number $\overline{\mathcal{M}}_{1}=0.100$, dotted line --- Mach number $\overline{\mathcal{M}}_{1}=0.050$ and solid line - Mach number $\overline{\mathcal{M}}_{1}=0.025$. All curves correspond to the semi-analytic method. Circles $\circ$ give the analytic solution for low frequencies Eq. $(34,35)$.

the indirect combustion noise is globally in the same range of magnitude as the direct one; it can be even ten times greater in the most unfavorable case (low inlet Mach number and high outlet Mach number). The ratio between indirect and direct noise in Fig. 7 is plotted versus the dimension-less pulsation $\Omega$. The parameter $\Omega\left(\omega \ell_{n} / \bar{c}_{1}\right)$ corresponds to the "acoustically" reduced pulsation and quantifies the "acoustic" compactness of the nozzle. To be representative of the "entropy" compactness of the nozzle, this dimensionless pulsation simply needs to be divided by the Mach number at the nozzle inlet. That is to say that the nozzle is ten (inlet Mach number 0.1) to forty (inlet Mach number 0.025) times less compact from an entropy point of view than acoustically. For reduced pulsations going to zero (compact nozzle assumption), the numerical computations converge to the ratio $\eta$ calculated with the analytical relations for the nozzle established by Marble \& Candel. 
Figure 7 shows that the slope of $\eta$ for $\Omega=0$ is close to zero, so that the analytical expression remains valid (less than $20 \%$ error) up to $\Omega=0.2$ in most cases, that is to say even when the entropy wavelength is of the order of the nozzle length. The fully-analytic approach provides thus a good idea of what the indirect-to-direct ratio can be. This ratio calculated with the fully-analytic approach is plotted versus the nozzle inlet and outlet Mach numbers in Fig. 8. Nowadays, the overall pressure ratio at takeoff for commercial aero-engines ranges from

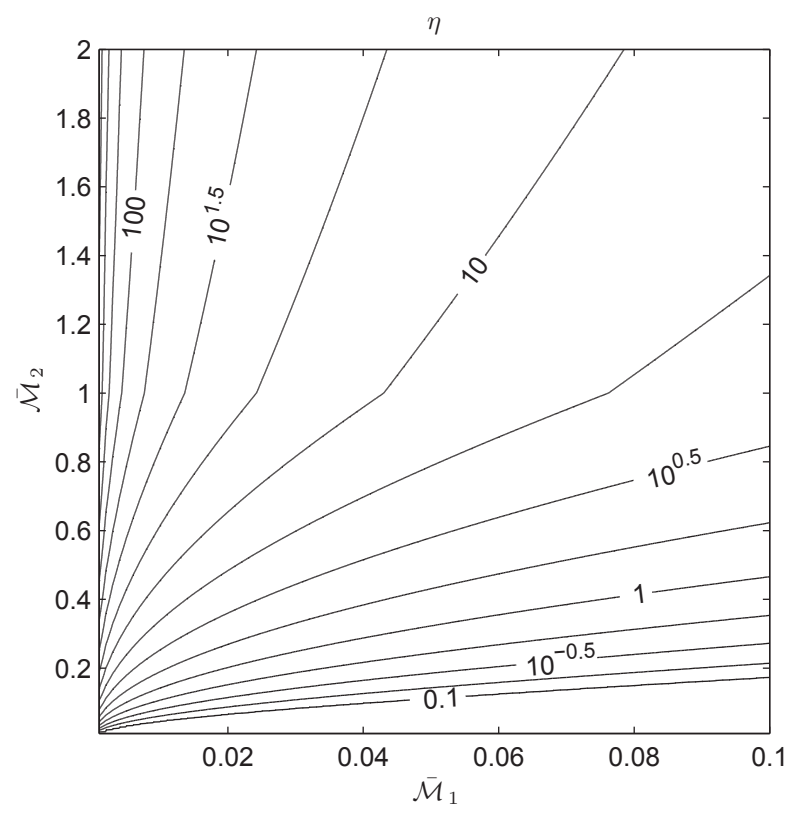

Figure 8. Estimation of the ratio $\eta$ between indirect and direct noise by the fully-analytic approach. The ratio $\eta$ is plotted here as a function of the Mach number $\overline{\mathcal{M}}_{1}$ representing the Mach number in the combustion chamber and at the nozzle inlet, and of the Mach number $\overline{\mathcal{M}}_{2}$ representing the outlet nozzle Mach number.

about 30 to 40, which is equivalent to an acceleration leading to an outlet Mach number of about 2.0. This graph shows that for an outlet Mach number between unity and two and an inlet Mach number close to 0.05 (condition which can be found in aero-engines) the indirect combustion noise can be more than ten times as important as the direct one. Equation (35) shows that when the outlet Mach number $\overline{\mathcal{M}}_{2}$ is high, the ratio $\eta$ depends only of the inlet Mach number $\overline{\mathcal{M}}_{1}$ and tends to $\left(1+\overline{\mathcal{M}}_{1}\right) / \overline{\mathcal{M}}_{1}(\gamma-1)$. With the previous value of the inlet Mach number of 0.05 , the maximum ratio $\eta$ is then greater than sixty. Of course the present estimation is quite oversimplified, since in a real engine, the strong azimuthal deviation of flow within the turbine stages have to be taken into account, as well as the blade loading and blade rows spacing. This approach has been followed by Cumpsty \& Marble, ${ }^{18,19}$ but the number of parameters involved in such a method is important and the results are enginedependent (even if general designs can be used to perform such a calculation). The present approach provides a simple method for the estimation of the indirect-to-direct noise ratio, 
and confirms the importance of indirect noise.

\section{Conclusion}

The noise produced by an aero-engine is generated either by the acoustic waves created by unsteady combustion (direct noise) or by the entropy waves created by combustion and convected through the turbine stages where they create noise (indirect noise). A simple quasi-1-D combustor model, based on a combustion chamber terminated by a nozzle has been used to evaluate all waves (acoustic and entropy) created by an unsteady flame zone and to quantify direct and indirect noise. Wave propagation in this model can be determined in the low frequency limit using fully analytical methods as suggested by Marble \& Candel or for all frequencies using a semi analytical-numerical technique where the wave propagation through the nozzle is solved using the Euler equations while the rest of the problem is handled analytically. Results demonstrate that the analytical approximation remains valid up to "acoustically" reduced pulsations of order of 0.2 , that is to say even when the entropy wavelength is of the order of the nozzle length, for the given range of inlet Mach numbers (0.025-0.100). They also show that the ratio of indirect to direct noise depends on two Mach numbers: the Mach number in the flame zone and the Mach number at the nozzle outlet. This ratio should be small for laboratory experiments but large in most real aero-engines, showing that research on combustion noise needs to incorporate indirect noise generation.

\section{Acknowledgments}

The authors gratefully acknowledge support from CINES for the access to computing resources. This work was partly funded by the FNRAE within the BRUCO Project. N. Lamarque is thanked for questions and discussions on theoretical backgrounds. F. Duchaine and A. Roux are thanked for their support about computational issues.

\section{References}

${ }^{1}$ Bragg, S., "Combustion noise," Journal of Institute of Fuel, Vol. 36, 1963, pp. 12-16.

${ }^{2}$ Strahle, W., "Combustion noise," Prog. Energy Comb. Sci., Vol. 4, 1978, pp. 157-176.

${ }^{3}$ Hassan, H. A., "Scaling of combustion-generated noise." J. Fluid Mech., Vol. 66, No. 3, 1974, pp. 445453.

${ }^{4}$ Ihme, M., Pitsch, H., and Bodony, D., "Radiation of Noise in Turbulent Non-Premixed Flames," In Proc. of the Combustion Institute, 2008.

${ }^{5}$ Candel, S., Durox, D., and Schuller, T., "Flame interactions as a source of noise and combustion instabilities." 10th AIAA/CEAS Aeroacoustics Conference - AIAA 2004-2928, 2004. 
${ }^{6}$ Candel, S., Durox, D., Ducruix, S., Birbaud, A.-L., Noiray, N., and Schuller, T., "Flame dynamics and combustion noise: progress and challenges," 11th CEAS-ASC Workshop of X3-Noise, Lisbon, Portugal, 2007.

${ }^{7}$ Crighton, D. G., Dowling, A. P., Williams, J. E. F., Heckl, M., and Leppington, F., Modern methods in analytical acoustics, Lecture Notes, Springer Verlag, New-York, 1992.

${ }^{8}$ Rajaram, R. and Lieuwen, T., "Parametric Studies of Acoustic Radiation from Turbulent Premixed Flames," Com. Sci. Tech., Vol. 175, No. 12, 2003, pp. 2269-2298.

${ }^{9}$ Rajaram, R. and Lieuwen, T., "Effect of Approach Flow Turbulence Characteristics on Sound Generation from Premixed Flames," 42nd AIAA Aerospace Sci. Meet. and Ex. - AIAA 2004-0461, 2004.

${ }^{10}$ Lieuwen, T., Mohan, S., Rajaram, R., and Preetham, "Acoustic radiation from weakly wrinkled premixed flames," Comb. Flame, Vol. 144, 2005, pp. 360-369.

${ }^{11}$ Tsien, H. S., "The transfer functions of rocket nozzles." J. Am. Rocket Soc., Vol. 22, 1952, pp. 139-143.

${ }^{12}$ Crocco, L., "Supercritical gaseaous discharge with high frequency oscillations." Aerotechnica, Vol. 33, 1953, pp. $46-53$.

${ }^{13}$ Candel, S., Analytical studies of some acoustic problems of jet engines., Ph.D. thesis, California Institute of Technology, Pasadena, California, 1972.

${ }^{14}$ Marble, F. E., Symposium on Transportation Noise, Stanford university, 1973.

${ }^{15}$ Marble, F. E. and Candel, S., "Acoustic disturbances from gas nonuniformities convected through a nozzle," J. Sound Vibration, Vol. 55, 1977, pp. 225-243.

${ }^{16}$ Ffowcs Williams, J. E. and Howe, M. S., "The generation of sound by density inhomogeneities in low Mach number nozzle flows," J. Fluid Mech., Vol. 70, 1975, pp. 605-622.

${ }^{17}$ Stow, S. R., Dowling, A. P., and Hynes, T. P., "Reflection of circumferential modes in a choked nozzle," J. Fluid Mech., Vol. 467, 2002, pp. 215-239.

${ }^{18}$ Cumpsty, N. and Marble, F., "The interaction of entropy fluctuations with turbine blade rows ; a mechanism of turbojet engine noise," Proc. R. Soc. Lond., Vol. 357, 1977, pp. 323-344.

${ }^{19}$ Cumpsty, N. and Marble, F., "Core noise from gas turbine exhausts," J. Sound Vibration, Vol. 54, 1977, pp. 297-309.

${ }^{20}$ Muthukrishnan, M., Strahle, W., and Neale, D., "Separation of hydrodynamic, entropy, and combustion noise in a gas turbine combustor," AIAA Journal, Vol. 16, No. 4, 1978, pp. 320-327.

${ }^{21}$ Bake, F., Michel, U., Rohle, I., Richter, C., Thiele, F., Liu, M., and Noll., B., "Indirect combustion noise generation in gas turbines," 11th AIAA/CEAS Aeroacoustics Conference - AIAA 2005-2830, 2005.

${ }^{22}$ Bake, F., Michel, U., and Rohle, I., "Investigation of Entropy Noise in Aero-Engine Combustors," Transaction of the ASME - 129, 2007.

${ }^{23}$ Bake, F., Kings, N., and Rohle, I., "Fundamental Mechanism of Entropy Noise in Aero-Engines: experimental investigation," J. Eng. Gas Turb. and Power, Vol. 130, 2008.

${ }^{24}$ Polifke, W., Paschereit, C., and Doebbeling, K., "Constructive and Destructive Interference of Acoustic and Entropy Waves in a Premixed Combustor with a Choked Exit," Int. J. Acoust. Vib., Vol. 6, 2001, pp. $135-146$.

${ }^{25}$ Eckstein, J., Freitag, E., Hirsch, C., and Sattelmayer, T., "Experimental study on the role of entropy waves in low-frequency oscillations for a diffusion burner," ASME Turbo Expo, edited by A. Paper, Vienna, Austria, 2004. 
${ }^{26}$ McManus, K., Poinsot, T., and Candel, S., "A review of active control of combustion instabilities," Prog. Energy Comb. Sci., Vol. 19, 1993, pp. 1-29.

${ }^{27}$ Dowling, A. P., "The calculation of thermoacoustic oscillations," J. Sound Vibration, Vol. 180, No. 4, 1995, pp. 557-581.

${ }^{28}$ Moase, W., Brear, M., and Manzie, C., "The forced response of choked nozzles and supersonic diffusers," J. Fluid Mech., Vol. 585, 2007, pp. 281-304.

${ }^{29}$ Schmitt, P., Poinsot, T., Schuermans, B., and Geigle, K., "Large-eddy simulation and experimental study of heat transfer, nitric oxide emissions and combustion instability in a swirled turbulent high pressure burner," J. Fluid Mech., Vol. 570, 2007, pp. 17-46.

${ }^{30}$ Poinsot, T. and Veynante, D., Theoretical and Numerical Combustion, R.T. Edwards, 2nd edition., 2005.

${ }^{31}$ Moureau, V., Lartigue, G., Sommerer, Y., Angelberger, C., Colin, O., and Poinsot, T., "Numerical methods for unsteady compressible multi-component reacting flows on fixed and moving grids," J. Comput. Phys., Vol. 202, No. 2, 2005, pp. 710-736.

${ }^{32}$ Childers, D. G., Modern Spectrum Analysis, IEEE Press, USA, 1978. 